\title{
The Mediator Chromatic Number of Grid Graphs
}

\author{
N. Roopesh \\ Research scholar \\ Department of Mathematics \\ Kongunadu Arts and Science College \\ Coimbatore - 641029
}

\author{
K.Thilagavathi \\ Assoc. Prof. of Mathematics \\ Department of Mathematics \\ Kongunadu Arts and Science College \\ Coimbatore - 641029
}

\begin{abstract}
In this paper, we determine the mediator chromatic number of cartesian product of a path with $K_{2}$ and thus prove that the mediator chromatic number of Ladder graphs and Grid graphs are polynomially solvable.
\end{abstract}

\section{General Terms}

Graph colouring, chromatic number, operations on graphs.

\section{Keywords}

Mediator colourclass, mediator colouring, mediator chromatic number.

\section{INTRODUCTION}

In graph theory, graph colouring is an assignment of colours to certain objects in a graph. Such objects can be vertices, edges, faces or a mixture of those. Among these, vertex colouring is the important kind. It is a usual custom to use the numbers $1,2,3, \ldots, k$ to colour a graph instead of the actual colours. A $k$-vertex colouring of a graph $G$ is an assignment of $k$ colours to the vertices of $G$ and it is proper if no two distinct adjacent vertices have the same colour. In this entire paper by colouring, we mean the proper vertex colouring. The mediator chromatic number [7] was introduced by N.Roopesh and K. Thilagavathi. They [8] shown that it can be used as a tool for checking the minimum of a harmonious colouring.

\section{DEFINITIONS $[7,8]$}

\subsection{Mediator Colouring Partition}

Let $G(V, E)$ be a simple graph and $\Pi=\left\{C_{1}, C_{2}, C_{3}, \ldots, C_{k}\right\}$ be a proper colouring partition of $V(G)$. This partition is said to be a mediator colouring partition if the following condition is satisfied.

(i) $C_{i} \cup C_{j}, i \neq j$ is not independent

or

(ii) There exist at least two edges from $C_{i} \cup C_{j}$ to some other colour class $C_{l}, l \neq i, j$

Note that the second choice ensures at least two edges incident to the vertex which is coloured as $c_{l}$ and the other ends with colours $c_{i}$ and $c_{j}$

\subsection{Mediator colouring}

Mediator colouring is a proper vertex colouring in which the colouring partition is a mediator colouring partition.

\subsection{Mediator chromatic number}

The maximum cardinality of a mediator colouring partition of a graph $G$ is called the mediator chromatic number of the graph. And it is denoted by $\chi_{M}(G)$.

Note : It can be noted that if the first condition of mediator colouring alone is satisfied for every $i$ and $j$, then it is called the complete colouring or achromatic colouring and in this case $\chi_{M}$ will become $\psi$.
Note: We denote the colour classes of the vertices with colour $c_{i}$ as $C_{i}$. Then two colour classes $C_{i}$ and $C_{j}$ are adjacent if there exist an edge with end points coloured as $c_{i}$ and $c_{j}$.

\subsection{Mediator set}

In a mediator colouring, any set $C_{l}$, such that there exists at least two edges from $C_{i} \cup C_{j}$ to $C_{l}$ (where $l \neq i, j$ ) is called a mediator colour class of $C_{i}, C_{j}$ and $c_{l}$ is called a mediator of $c_{i}, c_{j}$. The collection of all mediator colour classes is called the mediator set of the mediator colouring.

\section{MEDIATOR COLOURING OF CARTESIAN PRODUCT OF $P_{\boldsymbol{n}}$ WITH $\boldsymbol{K}_{2}$}

In graph theory, the Cartesian product [10] $G \times H$ of graphs $G$ and $H$ is a graph defined as follows : vertex set of $G \times H$ is the Cartesian product $V(G) \times V(H)$ and any two vertices $\left(u, u^{\prime}\right)$ and $\left(v, v^{\prime}\right)$ are adjacent in $G \times H$ if and only if either $u=v$ and $u^{\prime}$ is adjacent with $v^{\prime}$ or $u^{\prime}=v^{\prime}$ and $u$ is adjacent with $v$.

\subsection{Theorem}

For a path $P_{n}$ of odd length $n>1, \chi_{M}\left(P_{n} \times K_{2}\right)=n+m+2$ where $n=2 m+1$.

Proof

Consider a path $P_{n}$ of odd length $n$ and a complete graph $K_{2}$. Let $V\left(P_{n}\right)=\left\{v_{1}, v_{2}, \ldots, v_{n}, v_{n+1}\right\}$ and $V\left(K_{2}\right)=\left\{u_{1}, u_{2}\right\}$.Then $V\left(P_{n} \times K_{2}\right)=\left\{w_{1}, w_{2}, w_{3}, \ldots, w_{n+1}, x_{1}, x_{2}, x_{3}, \ldots, x_{n+1}\right\}$, where $w_{i}=\left(v_{i}, u_{1}\right)$ and $x_{i}=\left(v_{i}, u_{2}\right)$. Consider the colour class $C=\left\{c_{1}, c_{2}, c_{3}, \ldots, c_{n+m+2}\right\}$, assign the colours to the vertices of $P_{n} \times K_{2}$ as follows

Colour the vertices $w_{1}$ as $c_{1}, x_{1}, w_{2}, w_{2+4 k}$ and $x_{4 r}$ as $c_{2}$, where $k, r=1,2,3, \ldots,\left[\frac{n}{4}\right]$. Let $W^{\prime}=\left\{w_{i}\right\}-\left\{w_{1}, w_{2}, w_{2+4 k}\right\}$. Form a sequence $\left\{t_{i}\right\}, t_{i} \in W^{\prime}$ such that $\{i\}$ is a strictly increasing sequence. Consider $X^{\prime}=\left\{x_{i}\right\}-\left\{x_{1}, x_{4 r}\right\}$. Form a sequence $\left\{y_{h}\right\}, y_{h} \in X^{\prime}$ and $\{h\}$ is a strictly increasing sequence. Now for $i=1,2,3, \ldots, n-\left[\frac{n}{4}\right]-1$ and $h=$ $1,2,3, \ldots, n-\left[\frac{n}{4}\right]$ assign the colour $c_{2 i+2}$ to $t_{i}$ and $c_{2 h+1}$ to $y_{h}$. Then this colouring is a mediator, for the set of vertices coloured as $c_{2}$ will act as the colour class $C_{l}$ such that for each $i, j, i, j \neq l$ there exists at least two edges from $C_{i} \cup C_{j}$ to $C_{l}$. More over, it is the maximum colouring. For, suppose not, that is a mediator colouring is possible with a colour class $C^{\prime}$ with $\left|C^{\prime}\right|=|C|+1$. Let $c$ be the new colour added to $C$. Now to get such a colouring, only possibility is to recolour any of the vertex coloured as $c_{2}$. That is we have to consider the recolouring of the vertces of the following forms.

(i) $w_{2+4 k}, k=1,2,3, \ldots,\left[\frac{n}{4}\right]$
(ii) $x_{4 r}, r=1,2,3, \ldots,\left[\frac{n}{4}\right]$ 
(iii) $x_{1}, w_{2}$

Case 1

Suppose we are recolouring the vertex of the form $w_{2+4 s}$, $1 \leq s \leq\left[\frac{n}{4}\right]$, then $w_{2+4 s}$ is adjacent with at least two vetices of the graph. Consider any one such vertex say $w$. Now let $z \in N(w)$, and $a \in N(Z), a \neq w$.

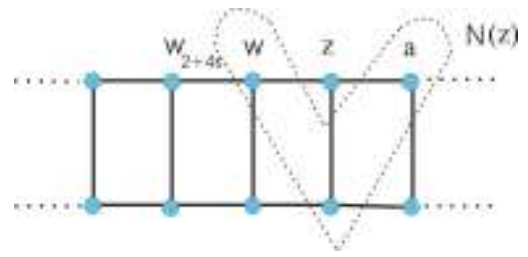

Figure 1: showing the selection of $a$

Consider the colouring map $\square: V \rightarrow C^{\prime}$ then $\left\{\square\left(w_{2+4 s}\right)\right\}$ $\cup\{\square(a)\}$ is independent. Then to make this colouring mediator, we need a different colour class $C_{l}$ such that there exists at least two edges from $\left\{\square\left(w_{2+4 s}\right)\right\} \cup\{\square(a)\}$ to $C_{l}$. Since we are going for a maximum colouring, the only way is to recolour the neighbour of $a$, which is coloured as $c_{2}$ to $c$. Then again using the previous arguement, we can find a vertex $b$ such that $\left\{\square\left(w_{2+4 s}\right)\right\} \cup\{\square(b)\}$ is independent. Thus, the recolouring process of $c_{2}$ to $c$ will continue upto the last vertex with colour $c_{2}$. Now the colour class will become $C^{\prime}-\left\{c_{2}\right\}$. Thus $\left|C^{\prime}\right|=|C|+1-1=|C|$, which is a contradiction. Therefore $C$ is the maximum colour class.

Case 2

If we are recolouring the vertex of the form $x_{4 s}, 1 \leq s \leq$ $\left[\frac{n}{4}\right]$,by using a similar arguement used in case 1 , we can prove that $C$ is the maximum colour class possible.

\section{Case 3}

If we are recolouring $x_{1}$ and $w_{2}$, we can easily see that the vertices $w_{3}, w_{4}, \ldots, w_{n+1}, x_{4}, x_{5}, \ldots, x_{n+1}$ are not adjacent with $x_{1}$ or $w_{2}$. Thus, again we can choose a vertex $a$ such that $\{c\} \cup\{\square(a)\}$ is independent, then we can proceed as in case 1 to prove the colour class $C$ is the maximum one.

It can be noted that any recolouring of the combination of the vertices amoung (i), (ii) and (iii) will also enable us to select a vertex $a$ such that $\{c\} \cup\{\square(a)\}$ is independent.

Thus in all the cases, we get the colourclass $C$ is the maximum one possible.

Hence for odd $n, \chi_{M}\left(P_{n} \times K_{2}\right)=n+m+2$ where $n=$ $2 m+1$.

\section{Example}

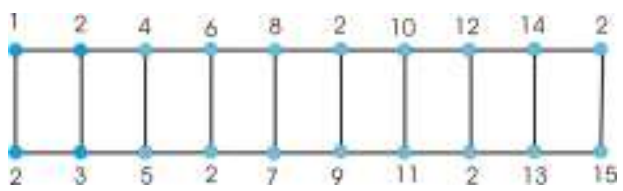

Figure 2: $\chi_{M}\left(P_{9} \times K_{2}\right)=9+4+2=15$

\subsection{Note}

For $n=1, \chi_{M}\left(P_{1} \times K_{2}\right)=4$

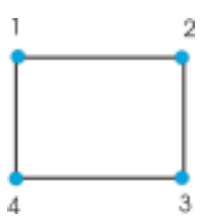

Figure 3: $\chi_{M}\left(P_{1} \times K_{2}\right)=4$

\subsection{Theorem}

For a path $P_{n}$ of even length, $\chi_{M}\left(P_{n} \times K_{2}\right)=n+\frac{n}{2}+2$.

Proof

Consider a path $P_{n}$ of even length $n$ and a complete graph $K_{2}$. Let $V\left(P_{n}\right)=\left\{v_{1}, v_{2}, v_{3}, \ldots, v_{n}, v_{n+1}\right\}$ and $V\left(K_{2}\right)=\left\{u_{1}, u_{2}\right\}$. Then

$V\left(P_{n} \times K_{2}\right)=\left\{w_{1}, w_{2}, w_{3}, \ldots, w_{n+1}, x_{1}, x_{2}, x_{3}, \ldots, x_{n+1}\right\}$,

where $w_{i}=\left(v_{i}, u_{1}\right)$ and $x_{i}=\left(v_{i}, u_{2}\right)$. Consider the colour class $C=\left\{c_{1}, c_{2}, c_{3}, \ldots, c_{n+\frac{n}{2}+2}\right\}$, and assign the colours to the vertices of $P_{n} \times K_{2}$ as follows.

Case 1: $n$ is a multiple of 4 .

Colour the vertices $w_{1}$ as $c_{1}$ and each vertex of the form $w_{3+4 k}$ or $x_{1+4 r}$ as $c_{2}$, where $k=0,1,2, \ldots, \frac{n}{4}-1$ and $r=$ $0,1,2, \ldots, \frac{n}{4} . \quad$ Let $\quad W^{\prime}=\left\{w_{1}, w_{2}, \ldots, w_{n+1}\right\} \quad-\left(\left\{w_{1}\right\} \cup\right.$ $\left.\left\{w_{3+4 k}: k=0,1,2, \ldots, \frac{n}{4}-1\right\}\right)$. Let $\left(t_{i}\right)_{i=1}^{\frac{3 n}{4}}$ be the sequence of elements from $W^{\prime}$ in order of increasing index.

Let $X^{\prime}=\left\{x_{1}, x_{2}, \ldots, x_{n+1}\right\}-\left(\left\{x_{1+4 r}: r=0,1,2, \ldots, \frac{n}{4}\right\}\right)$. Let $\left(y_{h}\right)_{h=1}^{\frac{3 n}{4}}$ be the sequence of elements from $X^{\prime}$ in order of increasing index. Now for $i, h=1,2,3, \ldots, \frac{3 n}{4}$ assign the colour $c_{2 i+2}$ to $t_{i}$ and $c_{2 h+1}$ to $y_{h}$.

Case 2: $n$ is not a multiple of 4 .

Colour $w_{1}$ as $c_{1}$ and each vertex of the form $w_{3+4 k}$ or $x_{1+4 r}$ as $c_{2}$, where $k, r=0,1,2, \ldots,\left[\frac{n}{4}\right]$. Similar to $X^{\prime}$ consider the set $W^{\prime}$,

$W^{\prime}=\left\{w_{1}, w_{2}, \ldots, w_{n+1}\right\}-\left(\left\{w_{1}\right\} \cup\left\{w_{3+4 k}: k=0,1,2\right.\right.$,

$\left.\left.\ldots,\left[\frac{n}{4}\right]\right\}\right)$. Let $\left(t_{i}\right)_{i=1}^{\frac{3 n}{4}}$ be the sequence of elements from $W^{\prime}$ in order of increasing index. Let $X^{\prime}=\left\{x_{1}, x_{2}, \ldots, x_{n+1}\right\}$ $-\left(\left\{x_{1+4 r}: r=0,1,2, \ldots,\left[\frac{n}{4}\right]\right\}\right) . \quad$ Let $\quad\left(y_{h}\right)_{h=1}^{\left[\frac{3 n}{4}\right]+1}$ be the sequence of elements from $X^{\prime}$ in order of increasing index. Now for $i=1,2,3, \ldots,\left[\frac{3 n}{4}\right]$ and $h=1,2,3, \ldots,\left[\frac{3 n}{4}\right]+1$ assign the colour $c_{2 i+2}$ to $t_{i}$ and $c_{2 h+1}$ to $y_{h}$.

Then this colouring is a mediator, for the set of vertices coloured as $c_{2}$ will act as the colour class $C_{l}$ such that for each $i, j$ such that $i, j \neq l$ there exists at least two edges from $C_{i} \cup C_{j}$ to $C_{l}$. That is, $\left\{C_{2}\right\}$ will act as the mediator set. Moreover, it is the maximum colouring. For suppose not, that is, a mediator colouring is possible with a colour class $C^{\prime}$ with $\left|C^{\prime}\right|=|C|+1$. Let $C^{\prime}=C \cup\{c\}$. To get such a colouring, the only possibility is to recolour the $c_{2}$ coloured vertices as $c$. That is, we have to consider the recolouring of some vertices of the following forms.

(i) $\mathrm{w}_{3+4 \mathrm{k}}$, where $k=\left\{\begin{array}{c}0,1,2, \ldots, \frac{n}{4}-1, \text { if } \mathrm{n} \equiv 0(\bmod 4) \\ 0,1,2, \ldots,\left[\frac{n}{4}\right], \text { otherwise }\end{array}\right.$

(ii) $\mathrm{x}_{1+4 \mathrm{r}}$, where $r=\left\{\begin{array}{c}0,1,2, \ldots, \frac{n}{4}, \text { if } \mathrm{n} \equiv 0(\bmod 4) \\ 0,1,2, \ldots,\left[\frac{n}{4}\right], \text { otherwise }\end{array}\right.$ 
Case $(i)$

Suppose we are recolouring some vertex $w$ of the form $w_{3+4 k}$, then it is adjacent to atleast two vertices of the graph. Let $z \in N(w)$, and $a \in N(z), a \neq w$. Consider the colouring map $\square: V \rightarrow C^{\prime}$ then $C_{\square(w)} \cup C_{\square(a)}$ is independent, where $C_{d}$ is the colour class corresponding to colour $d$. Then to make this colouring a mediator, we need a colour class $C_{l}$ such that there exists atleast two edges from $C_{\square(w)} \cup C_{\square(a)}$ to $C_{l}$. Since we are going for a maximum colouring, the only way is to recolour the neighbour of $a$, which is coloured as $c_{2}$ to $c$. Now starting with either vertex $w$ or $w^{\prime}$ ( the recoloured vertex), and again using the previous argument, we can find another vertex $b$ such that $C_{\square(w)} \cup C_{\square(b)}$ is independent. Thus, the recolouring process from $c_{2}$ to $c$ will continue upto the last vertex with colour $c_{2}$, which results in expelling the colour $c_{2}$ from the colour class $C^{\prime}$. Thus, $\left|C^{\prime}\right|=|C|+1-1=|C|$, which is a contradiction. Therefore, $C$ is the maximum colour class.

Case (ii)

If we are recolouring some vertex of the form $x_{4 r+1}$, by a similar arguement as Case $(i)$, we can prove that $C$ is the maximum colour class possible.

It can be noted that any recolouring of the combination of vertices in case ( $i)$ and case (ii) will also enable us to select a vertex $a$ such that $C_{c} \cup C_{\square(a)}$ is independent, or in expelling one already existing colour and thus reducing the cardinality of the new colour class by one.

Thus in all the cases $C$ is the maximum colour class possible. Hence for even $n, \chi_{M}\left(P_{n} \times K_{2}\right)=n+\frac{n}{2}+2$

\section{CONCLUSION}

The graph $P_{n} \times K_{2}$ is generally known as the ladder graph $L_{n+1}$. Also it is equivalent to the grid graph

$G_{2, n+1}$. By theorem 3.1 , for odd $n$ the mediator chromatic number of $L_{n+1}$ and $G_{2, n+1}$ is $n+m+2$ where $n=2 m+1$. For even $n$, the mediator chromatic number of $L_{n+1}$ and $G_{2, n+1}$ is given by theorem 3.2 as $n+\frac{n}{2}+2$.

Thus, we conclude that for any $n>1, \chi_{M}\left(P_{n} \times K_{2}\right)=n+$ $\left[\frac{n}{2}\right]+2$.
Hence, $\chi_{M}\left(L_{n+1}\right)=\chi_{M}\left(G_{2, n+1}\right)=n+\left[\frac{n}{2}\right]+2$.

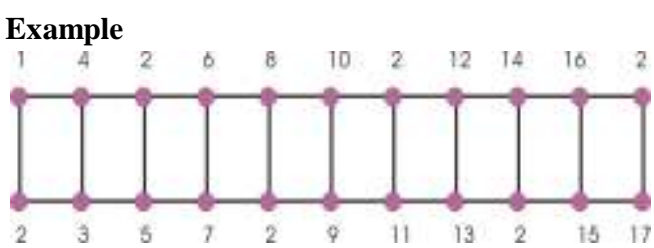

Figure 4: $\chi_{M}\left(P_{10} \times K_{2}\right)=10+5+2=17$

\section{REFERENCES}

[1] J. A Bondy and U.S.R. Murty, Graph theory with Applications. London: MacMillan (1976).

[2] Frank Harary and Stephen Hedetniemi, The Achromatic number of a graph. Journal of Combinatorial Theory, 8 (1970) 154-161.

[3] Frank Harary, Graph Theory. Narosa Publishing Home (1969).

[4] Frank Harary, Stephen Hedetniemi and Geert Prins An interpolation theorem for graphical homomorphisms. Portugaliae Mathematica, 26-Fasc.4 (1967).

[5] M. Farber, G. Hahn, P. Hell and D. J Miller Concerning the achromatic number of graphs. J. Combinatorial Theory, Ser. B, 40 (1986) 21-39.

[6] Gary Chartrand and Ping Zhang Chromatic graph theory. CRC Press (2009).

[7] N. Roopesh and K. Thilagavathi, Mediator colouring of graphs . Far East Journal of Applied Mathematics,(submitted).

[8] N. Roopesh and K. Thilagavathi, Relation between Harmonious colouring and Mediator colouring. Applied Mathematics E-Notes, (communicated)

[9] N. Roopesh and K. Thilagavathi, Mediator colouring of certain product of a path with $K_{2}$. Proceedings of the International Conference on Mathematics and Computer Science (ICMCS) (2010), 115-118.

[10] Yukio Shibata and Yosuke Kikuchi, Graph products based on the distance in graphs. IEICE Trans. Fundamentals, E83-A. No.3 (March 2000) 459-464. 